\title{
Engineering a Cytidine Aminotransferase for Biocatalytic Production of the Antiviral Molnupiravir
}

Ashleigh J. Burke ${ }^{[a]}$, William R. Birmingham ${ }^{[a]}$, Ying Zhuo ${ }^{[a]}$, Bruna Zucoloto da Costa ${ }^{[a]}$, Rebecca Crawshaw $^{[a]}$, Thomas W. Thorpe ${ }^{[a]}$, Ian Rowles ${ }^{[a]}$, James Finnigan ${ }^{[b]}$, Carl Young ${ }^{[b]}$, Simon J. Charnock ${ }^{[b]}$, Sarah L. Lovelock ${ }^{[a]}{ }^{*}$, Nicholas J. Turner ${ }^{[\mathrm{a}]^{*}}$ and Anthony P. Green ${ }^{[\mathrm{a}]^{*}}$

[a] Department of Chemistry, University of Manchester, Manchester Institute of Biotechnology, 131 Princess Street, Manchester, M1 7DN, UK

[b] Prozomix Ltd, Building 4, West End Ind. Estate, Haltwhistle, UK, NE49 9HA.

\begin{abstract}
The COVID-19 pandemic highlights the urgent need for cost-effective processes to rapidly manufacture antiviral drugs at scale. Here we report a concise biocatalytic process for Molnupiravir, a nucleoside analogue currently in phase 3 clinical trials as an orally available treatment for SARS-CoV2. Key to the success of this process was the development of a cytidine aminotransferase for the production of $\mathrm{N}$-hydroxy-cytidine through evolutionary adaption of the hydrolytic enzyme cytidine deaminase. This engineered biocatalyst performs $>100,000$ turnovers in less than 30 minutes, operates at $180 \mathrm{~g} / \mathrm{L}$ substrate loading and benefits from in situ crystallization of the $N$-hydroxy-cytidine product ( $>90 \%$ yield), which can be converted to Molnupiravir by a selective $5^{\prime}$-acylation using Novozym ${ }^{\circledR} 435$.
\end{abstract}

\section{Introduction}

Molnupiravir 1 is a nucleoside analogue currently undergoing clinical trials for the treatment of COVID19 (Figure 1).[1] The structural simplicity of Molnupiravir compared to alternative therapies (e.g. Remdesivir) combined with its oral availability and broad-spectrum antiviral activities make it an attractive candidate for the global treatment of viral infections. In view of the urgent need to develop therapies for treating the Covid-19 pandemic there has been considerable interest in developing synthetic routes to this API. The original synthesis of 1 required 10 steps and proceeded in $<10 \%$ overall yield.[2] Subsequently Vasudevan et al., reported a more streamlined approach starting from either cytidine 2 or its 5-O-butyryl derivative.[3] Steiner et al., chose to start from uridine and modified the method for introduction of the $\mathrm{N}$-hydroxy unit into the nucleobase by activation via a triazolyl derivative.[4] Very recently the Merck group disclosed an elegant chemoenzymatic approach starting from D-ribose which involved introduction of the uracil base using a cocktail of four enzymes.[5]

We sought to develop an efficient and sustainable route for the biocatalytic preparation of 1 that can be implemented on scale. All previous syntheses elected to install the $N$-hydroxy unit using chemical methods whereas we envisioned the direct conversion of cytidine $\mathbf{2}$ to $\mathbf{N}$-hydroxy-cytidine $\mathbf{4}$ using a biocatalyst. Since $\mathrm{N}$-hydroxy-cytidine $\mathbf{4}$ had previously been shown to undergo lipase catalysed acylation at $\mathrm{C}-5-\mathrm{OH},[3]$ we viewed this approach as a direct biocatalytic process to Molnupiravir $\mathbf{1}$ from readily available starting materials. Our desired route would require a cytidine aminotransferase capable of selectively installing the $\mathrm{N}$-hydroxy unit from hydroxylamine in a bulk water phase. At the outset of the project there was no known enzyme for this transformation. However, we were intrigued by a report which demonstrated that $\mathrm{N}$-hydroxy-cytidine $\mathbf{4}$ could be hydrolysed to uridine $\mathbf{3}$ by wildtype cytidine deaminase, albeit at a very low rate (ca. $2 \%$ of activity compared to cytidine 2).[6] Cytidine deaminase (CD; EC 3.5.4.5) is a zinc containing hydrolytic enzyme which catalyses the 
conversion of cytidine $\mathbf{2}$ to uridine $\mathbf{3}$ using water.[7] The enzyme is widely distributed amongst organisms and is involved in the salvage of cytidine for uridine mono-phosphate synthesis. Interestingly wild-type cytidine deaminase from $E$. coli has been used on scale for hydrolysis of the nucleoside analogue 2'-deoxy-3'-thiacytidine and hence it appeared to be an attractive choice as a potential industrial biocatalyst.[8] On the basis of these observations we initiated experiments aimed at converting cytidine $\mathbf{2}$ to $\mathrm{N}$-hydroxy-cytidine $\mathbf{4}$ catalysed by CD.

a)<smiles>Nc1ccn([C@@H]2O[C@H](CO)[C@@H](O)[C@H]2O)c(=O)n1</smiles>

Cytidine 2

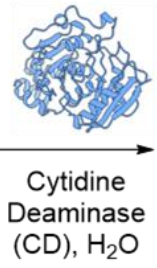

Directed

Evolution

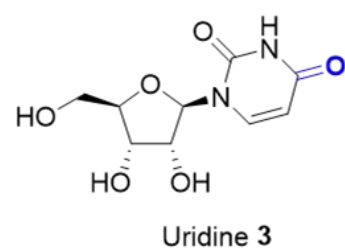

Uridine 3 b)

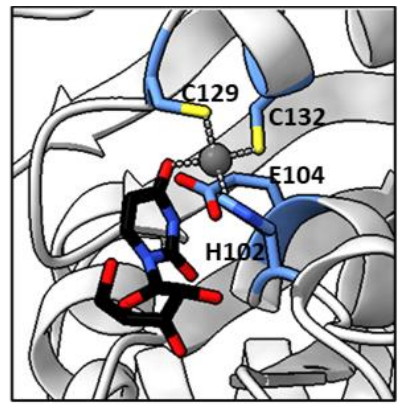

c)
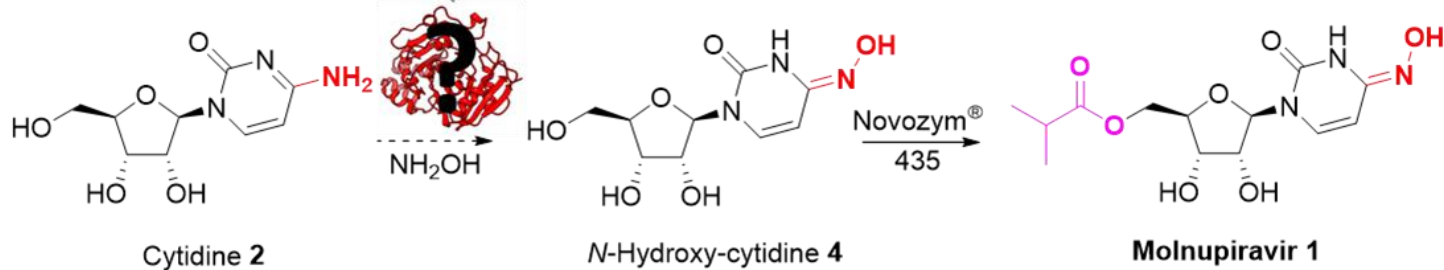

Molnupiravir 1

Figure 1. a) Wild-type cytidine deaminase (CD) catalyses the conversion of cytidine $\mathbf{2}$ to uridine $\mathbf{3}$ in the presence of water. $b$ ) The active site of CD with uridine bound (PDB code: 1AF2). The Zn ion is shown in grey. The Zn binding residues His102, Cys129 and Cys132, and catalytic Glu104 are shown as atom-coloured sticks with blue carbons. Uridine ligand is shown as atom-coloured sticks with black carbons. c) Proposed synthetic route to Molnupiravir $\mathbf{1}$. Cytidine $\mathbf{2}$ is converted to $\mathrm{N}$-hydroxy-cytidine 4 by an engineered cytidine aminotransferase followed by acylation using Novozym ${ }^{\circledR}-435$ to give Molnupiravir $1 .{ }^{[3]}$

\section{Results \& Discussion}

CD from E. coli was expressed in BL21(DE3) cells and purified to homogeneity via nickel affinity chromatography. To investigate whether this enzyme could serve as a biocatalyst for the preparation of $\mathrm{N}$-hydroxy-cytidine 4 , we initially established direct spectrophotometric assays to monitor the interconversion of cytidine $\mathbf{2}$, uridine $\mathbf{3}$ and $\mathrm{N}$-hydroxy-cytidine $\mathbf{4}$, based on diagnostic differences between the UV-Vis spectra of these species. Such assays are valuable to allow high-throughput and real-time analysis of biotransformations. In particular, the $\mathrm{N}$-hydroxy-cytidine $\mathbf{4}$ spectrum shows a diagnostic feature at $>310 \mathrm{~nm}$ that is not present in either cytidine $\mathbf{2}$ or uridine $\mathbf{3}$, thus allowing $\mathrm{N}$ hydroxy-cytidine $\mathbf{4}$ formation/decay to be easily monitored at these wavelengths.

We initially employed this assay to investigate the conversion of $N$-hydroxy-cytidine 4 to uridine $\mathbf{3}$. Consistent with previous reports, cytidine deaminase promotes $\mathrm{N}$-hydroxy-cytidine 4 hydrolysis under ambient conditions, with complete conversion of $100 \mu \mathrm{M}$ substrate achieved in 60 minutes $(0.1 \%$ enzyme loading, Figure $2 \mathrm{~b}$ ). Encouraged by these results, we next turned our attention to the more 
challenging synthesis of $\mathrm{N}$-hydroxy-cytidine $\mathbf{4}$ using either uridine $\mathbf{3}$ or cytidine $\mathbf{2}$ as a starting material. Pleasingly, biotransformations carried out in the presence of $5 \%$ hydroxylamine in water led to the accumulation of a product with spectral features consistent with $\mathrm{N}$-hydroxy-cytidine 4 . Similar final concentrations of $\mathbf{4}$ are formed using either $\mathbf{2}$ or $\mathbf{3}$ as a starting material, or in reactions starting from 4, suggesting that the enzyme establishes an equilibrium distribution of products (Figure 2b). As anticipated, the ratio of 4:3 formed is dependent on hydroxylamine concentration and 1:6 and 4:1 mixtures were achieved using $10 \%$ and $50 \%$ hydroxylamine solutions, respectively. Significantly, the concentration of product 4 does not accumulate beyond the equilibrium distribution irrespective of the hydroxylamine concentration used (Figure 2a), suggesting that cytidine hydrolysis followed by uridine amination (pathway B) outcompetes direct amine transfer (pathway A) with the wild type enzyme.

These results highlight biotransformations with cytidine deaminase as a promising strategy for the production of $\mathrm{N}$-hydroxy-cytidine 4. Unfortunately under thermodynamic control significant quantities of uridine by-product are formed, even at elevated hydroxylamine concentrations. Furthermore, the use of such high $\mathrm{NH}_{2} \mathrm{OH}$ concentrations is undesirable for large scale applications and leads to the formation of impurities over extended reaction times. To address these shortcomings, we elected to engineer $C D$ via directed evolution to optimize pathway A whilst minimizing pathway $B$. Similar strategies have been employed to control the partitioning of transglycosylation/hydrolysis by glycoside hydrolases.[9],[10] Accelerating the direct amination of cytidine in this way should allow accumulation of $\mathbf{4}$ under kinetic control at reduced $\mathrm{NH}_{2} \mathrm{OH}$ concentrations.

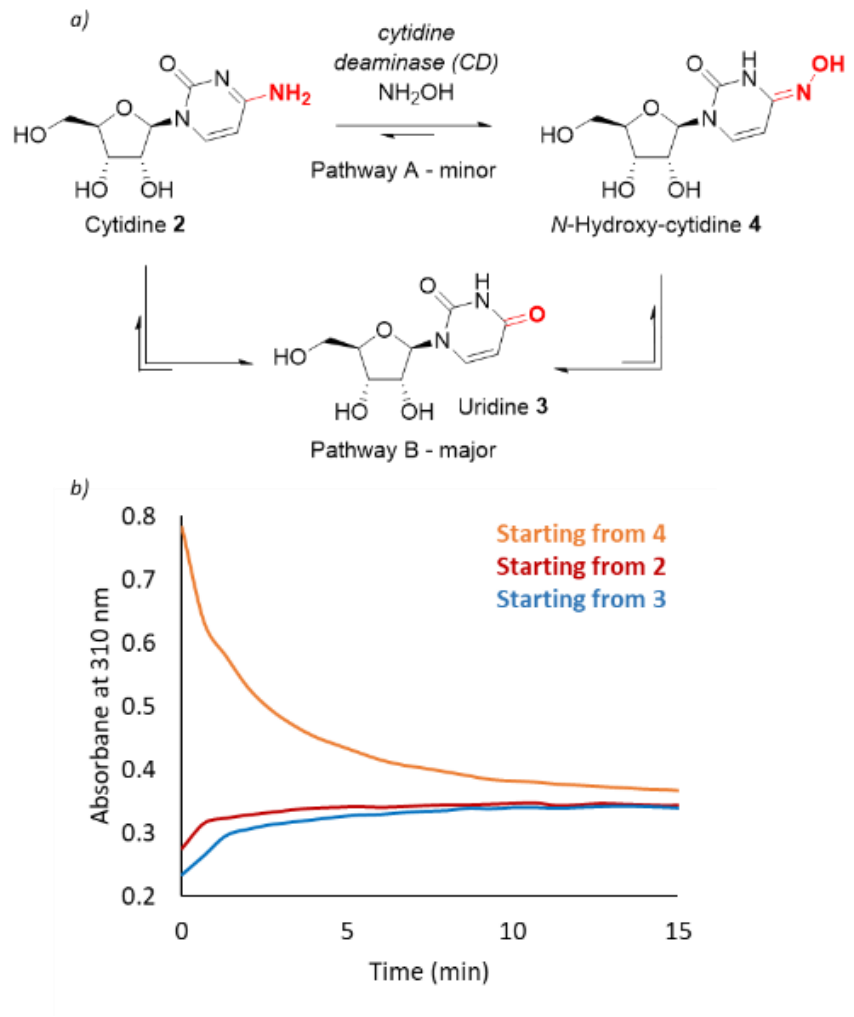

Figure 2. a) Pathways for conversion of cytidine $\mathbf{2}$ to $\mathrm{N}$-hydroxy-cytidine $\mathbf{4}$ catalyzed by cytidine deaminase. a) A minor pathway $\mathrm{A}$ involves direct conversion of $\mathbf{2}$ to $\mathbf{4}$ using $\mathrm{NH}_{2} \mathrm{OH}$ as a nucleophile. Pathway B involves initial hydrolysis of $\mathbf{2}$ to uridine 3 which is subsequently transformed to $\mathbf{4}$ through 
condensation with $\mathrm{NH}_{2} \mathrm{OH}$. Pathway $\mathrm{B}$ is the dominant pathway when using the wild-type enzyme, leading to an equilibrium distribution of 3 and 4 that is dependent on $\mathrm{NH}_{2} \mathrm{OH}$ concentration. b) The conversion of cytidine $2(1 \mathrm{mM})$ and uridine $\mathbf{3}(1 \mathrm{mM})$ to $N$-hydroxy-cytidine 4 by $\mathrm{CD}(5 \mu \mathrm{M})$ in the presence of $5 \% \mathrm{NH}_{2} \mathrm{OH}$ is monitored by increasing absorbance at $310 \mathrm{~nm}$. Similar final concentrations of $\mathrm{N}$-hydroxy-cytidine are formed using either $\mathbf{2}$ (red) or $\mathbf{3}$ (blue) as a starting material, or in reactions starting from $N$-hydroxy-cytidine 4 (orange).

To this end, iterative rounds of site saturation mutagenesis were performed using NNK degenerate primers, targeting residues within close proximity to the $\mathrm{Zn}$ cofactor and the substrate binding pocket. Beneficial diversity identified during rounds of evolution was combined by DNA shuffling. The aforementioned spectrophotometric assay was used to evaluate individual variants as crude cell lysates arrayed in 96-well plates, using $50 \mathrm{mM} 2$ as the substrate and $1 \% \mathrm{NH}_{2} \mathrm{OH}(\mathrm{pH}=7)$ as the reaction medium. Throughout evolution, we identified improved variants with kinetic profiles consistent with rapid initial accumulation of 4 beyond the equilibrium position followed by slower redistribution of products to equilibrium (Figure $3 b$ ).
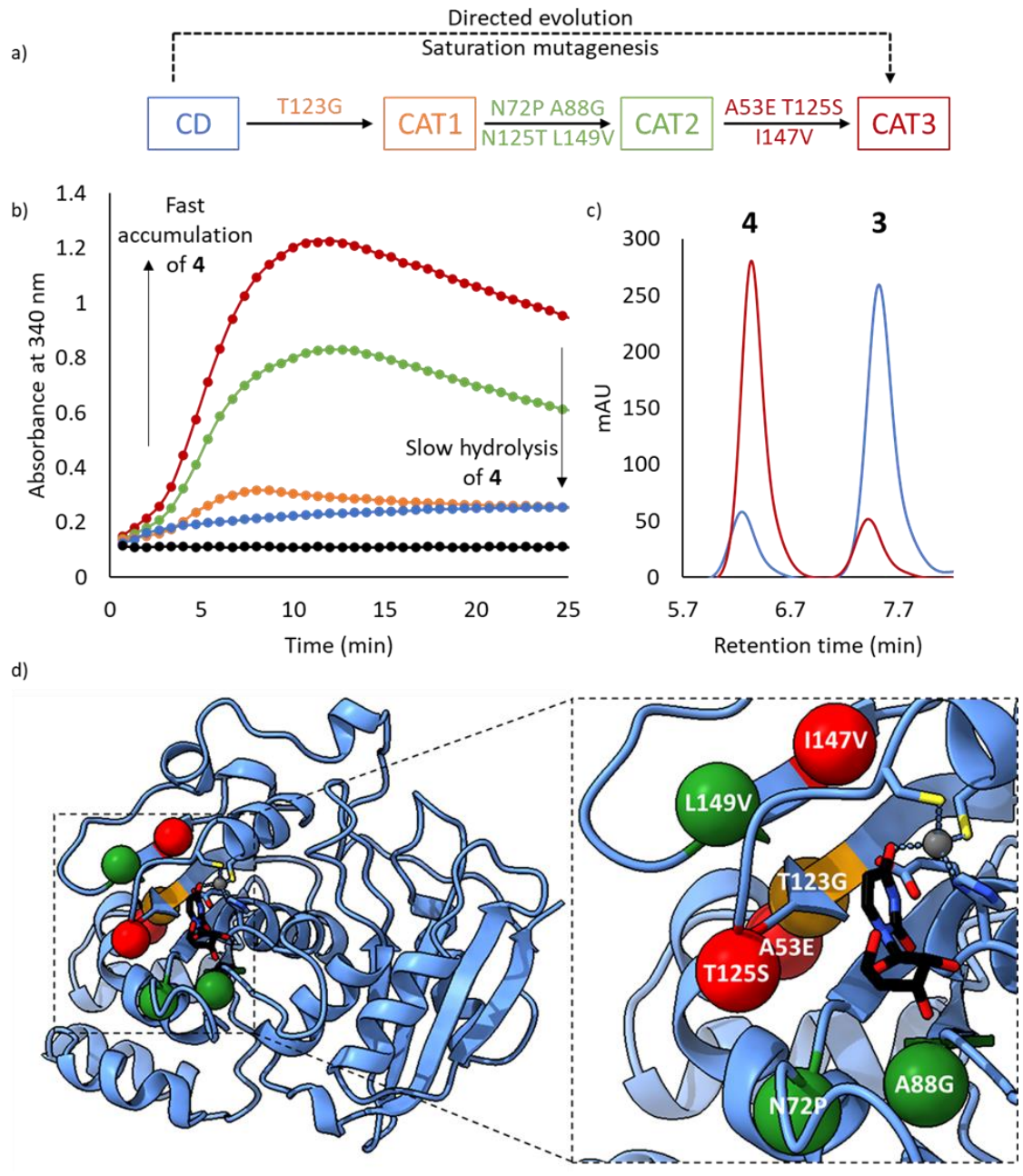

Figure 3. a) Directed evolution of $C D$ to CAT3 showing mutations installed during each round. b) Time course for the formation of $N$-hydroxy-cytidine $\mathbf{4}$ from cytidine $\mathbf{2}(50 \mathrm{mM})$ catalysed by CAT3 $(2.5 \mu \mathrm{M}$, red) CAT2 (2.5 $\mu \mathrm{M}$, green) CAT1 (2.5 $\mu \mathrm{M}$, orange) wild-type CD $(2.5 \mu \mathrm{M}$, blue) and no enzyme (black) in the presence of $1 \% \mathrm{NH}_{2} \mathrm{OH}, \mathrm{pH}=7$. The evolved $\mathrm{CD}$ variants show initial accumulation of $\mathrm{N}$-hydroxycytidine 4 beyond the equilibrium position followed by slower redistribution of products to 
equilibrium. c) HPLC traces showing cytidine $2(500 \mathrm{mM})$ conversion to $\mathrm{N}$-hydroxy-cytidine 4 and uridine 3 by CAT3 $\left(25 \mu \mathrm{M}\right.$, red) and wild-type $\mathrm{CD}(25 \mu \mathrm{M}$, blue $)$ in the presence of $10 \% \mathrm{NH}_{2} \mathrm{OH}, \mathrm{pH}=7$. Retention times of $\mathbf{3}$ and $\mathbf{4}$ are identical to authentic standards. d) The active site of CD with uridine bound (PDB code: $1 \mathrm{AF} 2$ ). Mutations installed in round 1 (T123G) are shown as orange spheres, round 2 (N72P A88G L149V) as green spheres, and round 3 (A53E T125S I147V) as red spheres. N125T from round 2 is not included as this was subsequently mutated in round 3 . The uridine ligand is shown as atom-coloured sticks with black carbons and the $\mathrm{Zn}$ cofactor is shown in grey. $\mathrm{Zn}$ binding residues His102,Cys 129 and Cys132, and catalytic Glu104 are shown as atom-coloured sticks with blue carbons.

The most promising variant to emerge following three rounds of evolution (CAT3) contains 7 mutations clustered around the active site. As intended CAT3 operates as an efficient aminotransferase, promoting the conversion of $\mathbf{2}$ to $\mathbf{4}$ even at low $\mathrm{NH}_{2} \mathrm{OH}$ concentrations and high substrate loadings. For example, at $500 \mathrm{mM} 2$ and $10 \% \mathrm{NH}_{2} \mathrm{OH}(\mathrm{pH} 7)$ CAT3 produces an 8:1 ratio of 4:3. In contrast, the wild-type enzyme produces a 1:6 ratio of 4:3 under identical conditions at equilibrium.

With a promising biocatalyst in hand, our attention turned to reaction intensification and product isolation. We observed that $\mathrm{N}$-hydroxy-cytidine is substantially less soluble than either cytidine or uridine in aqueous solutions, presenting an opportunity for dynamic crystallization of 4 to allow facile product isolation and further favour the distribution of 4:3. By increasing the substrate loading (750 $\mathrm{mM}$ ) and reducing the reaction temperature $\left(4^{\circ} \mathrm{C}\right)$ in situ crystallization of 4 was observed. Following optimisation of reaction conditions, 4 was isolated in $90 \%$ yield and $>97 \%$ purity in only 90 minutes using 0.003 mol\% CAT3 with no additional purification steps.

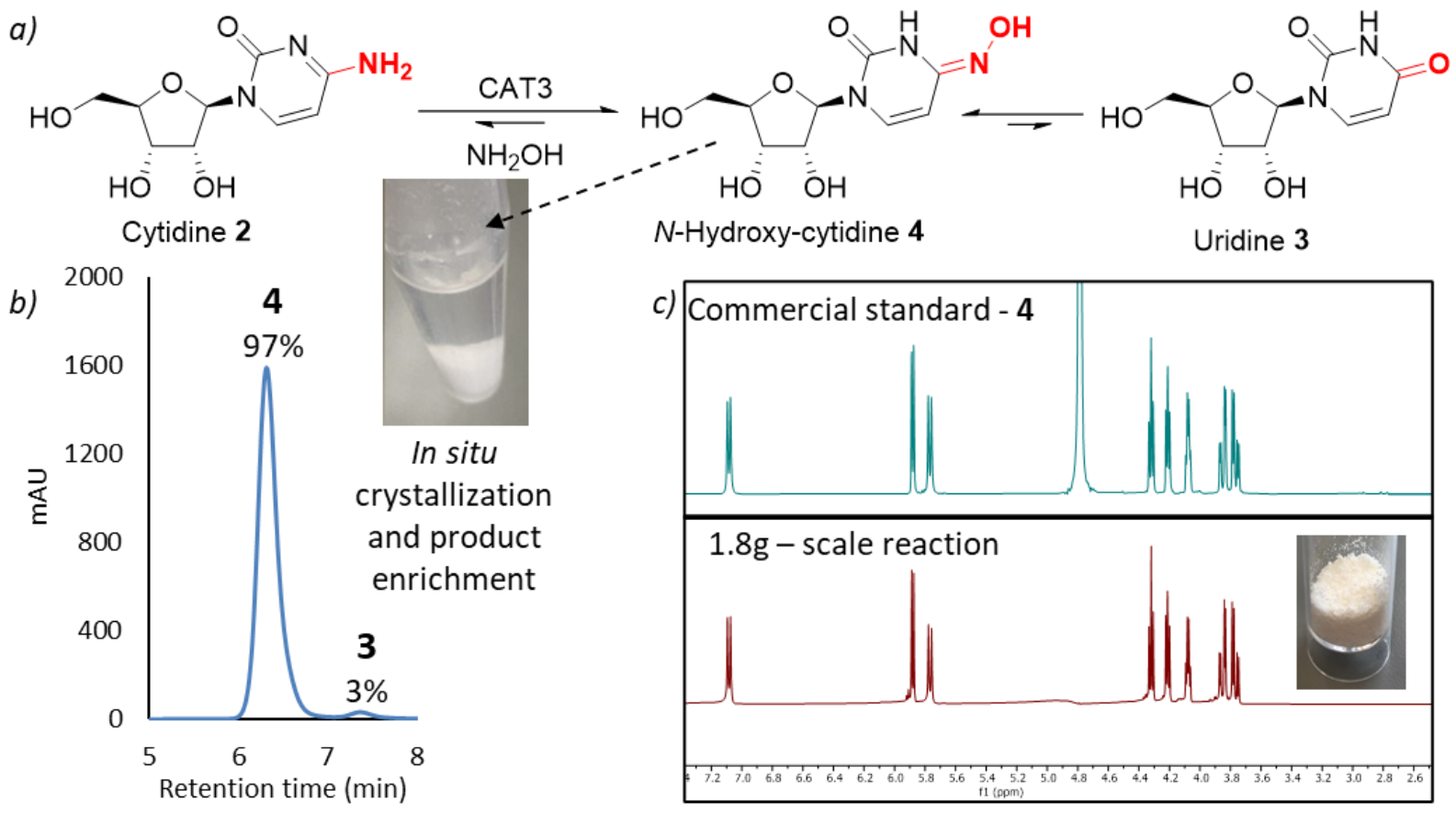

Figure 4. a) In situ crystallization of $\mathrm{N}$-hydroxy-cytidine 4 in reactions catalysed by CAT3 leads to product enrichment. Reaction conditions: $750 \mathrm{mM} \mathrm{2,10 \%} \mathrm{NH}_{2} \mathrm{OH}(\mathrm{pH}=7), 4^{\circ} \mathrm{C}$. b) HPLC trace of the 
product isolated from the biotransformation described in (a). $\mathrm{N}$-hydroxy-cytidine $\mathbf{4}$ is isolated in $90 \%$ yield and $>97 \%$ purity as confirmed by HPLC analysis and analysis of the ${ }^{1} \mathrm{H}$ NMR spectra. c) Stacked ${ }^{1} \mathrm{H}$ NMR traces of $\mathrm{N}$-hydroxy-cytidine 4, commercial standard (top) and the product isolated from the biotransformation described in (a) (bottom). Inset shows a picture of the isolated $N$-hydroxy-cytidine 4.

\section{Conclusions}

In summary, we have developed an efficient biocatalytic synthesis of $\mathrm{N}$-hydroxy-cytidine 4 , a key intermediate for the production of Molnupiravir 1 . The process takes advantage of an engineered cytidine aminotransferase and benefits from dynamic product crystallization to provide a scalable and sustainable manufacturing route to an important molecule in the fight against viral infections. Given that selective $5^{\prime}$-acylation of $N$-hydroxy-cytidine can be achieved with lipases[3], this work will contribute to an integrated biocatalytic strategy for Molnupiravir synthesis using cytidine as an inexpensive and abundant starting material.

\section{Acknowledgements}

We would like to thank the Bill \& Melinda Gates Foundation for funding and are particularly grateful to Dr. John Dillon, Dr. Trevor Laird and Dr. Silpa Sundaram for their helpful comments and advice during this project. We also acknowledge Dr Aisling Ní Cheallaigh for helpful discussions on product isolation.

\section{Selected Experimental}

DNA and protein sequence of CAT3

Mutations from wild-type CD: A53E N72P A88G T123G T125S I147V L149V

ATGCACCCGCGTTTTCAGACGGCGTTCGCCCAGTTAGCTGACAATCTGCAGAGTGCCTTGGAGCCTATCTTGG CAGATAAGTATTTTCCGGCCCTGCTGACCGGGGAGCAAGTTTCCTCCTTGAAAAGCGCTACGGGACTGGACGA AGATGCTCTTGAGTTTGCACTGCTTCCACTTGCTGCAGCTTGTGCACGTACCCCTTTGTCCAACTTCCCTGTCGG TGCGATTGCGCGTGGTGTAAGCGGAACCTGGTATTTCGGGGGCAACATGGAGTTTATCGGGGCGACTATGCA GCAAACCGTTCATGCTGAACAGAGCGCGATCAGCCATGCGTGGCTGAGCGGGGAGAAGGCATTAGCCGCGA TCGGGGTGAGTTACACGCCATGCGGACACTGTCGCCAGTTTATGAATGAGTTGAACTCAGGCTTGGATCTTCG TGTTCACGTGCCGGGACGTGAAGCCCATGCTTTACGTGACTACTTGCCTGATGCCTTCGGTCCCAAGGACTTA GAAATCAAGACCCTTCTTATGGACGAACAAGACCACGGATATGCGCTTACTGGAGATGCACTGTCCCAGGCTG CAATCGCTGCGGCCAACCGTAGCCACATGCCCTACTCCAAGAGTCCGAGCGGTGTCGCACTGGAGTGTAAAG ATGGACGTATTTTCAGCGGCTCATACGCGGAGAATGCGGCTTTCAATCCCACTCTTCCCCCGTTACAAGGAGC GCTGATTCTTTTAAATTTGAAGGGCTATGACTACCCAGACATTCAACGTGCAGTGCTTGCCGAGAAGGCGGAC GCACCATTGATCCAATGGGATGCTACCAGCGCAACTCTGAAAGCTTTAGGGTGTCACAGCATCGATCGCGTGC TGTTAGCCCTCGAGCACCACCACCACCACCAC

MHPRFQTAFAQLADNLQSALEPILADKYFPALLTGEQVSSLKSATGLDEDALEFALLPLAAACARTPLSNFPVGAIAR GVSGTWYFGGNMEFIGATMQQTVHAEQSAISHAWLSGEKALAAIGVSYTPCGHCRQFMNELNSGLDLRVHVPG REAHALRDYLPDAFGPKDLEIKTLLMDEQDHGYALTGDALSQAAIAAANRSHMPYSKSPSGVALECKDGRIFSGSY AENAAFNPTLPPLQGALILLNLKGYDYPDIQRAVLAEKADAPLIQWDATSATLKALGCHSIDRVLLALEHHHHHH 
Biocatalytic Synthesis of $N$-hydroxy-cytidine

Cytidine $(1.8 \mathrm{~g}, 750 \mathrm{mM},[180 \mathrm{~g} / \mathrm{L}])$ was dissolved in Hydroxylamine $(9 \mathrm{~mL}, 10 \%$ in water, $\mathrm{pH}=7)$ in a 50 $\mathrm{mL}$ falcon tube and cooled to $4{ }^{\circ} \mathrm{C}$. CAT3 purified enzyme ( $1 \mathrm{~mL}, 25 \mathrm{uM}, 0.7 \mathrm{~g} / \mathrm{L}$ final concentration) was added to the reaction mixture. The reaction was left on a tabletop roller for 90 minutes at $4{ }^{\circ} \mathrm{C}$ during which time the $\mathrm{N}$-Hydroxy-cytidine product crystallized in situ. The reaction mixture was centrifuged at $4000 \mathrm{rpm}$ for 5 minutes and the supernatant removed. The remaining solid was freeze dried overnight to give a free flowing white powder (1.71g, 90\% yield, $>97 \%$ purity).

\section{References}

[1] Cox, R. M.; Wolf, J. D.; Plemper, R. K. Nature Microbiology 2021, 6, 11-18.

[2] a) Painter, G. R., Bluemling, G. R.; Natchus, M. G.; Guthrie, D. WO2019113462, 2018. b) Painter, G. R.; Perryman, D.; Bluemling, G. R. WO2019173602, 2019.

[3] Vasudevan, N.; Ahlqvist, G. P.; McGeough, C. P.; Paymode, D. J.; Cardoso, F. S. P.; Lucas, T.; Dietz, J.-P.; Opatz, T.; Jamison, T. F.; Gupton, F. B.; Snead, D. R. Chem. Commun. 2020, 56, 13363-13364. b) Ahlqvist, G. P.; McGeough, C. P.; Senanayake, C.; Armstrong, J. D.; Yadaw, A.; Roy, S.; Ahmad, S.; Snead, D. R.; Jamison T. F. ACS Omega 2021, 6, 10396-10402.

[4] Steiner, A.; Znidar, D.; Ötvös, S. B.; Snead, D. R.; Dallinger, D.; Kappe, C. O. Eur. J. Org. Chem. 2020, 6736-6739.

[5] Benkovics, T.; McIntosh, J.; Silverman, S.; Kong, J.; Maligres, P.; Itoh, T.; Yang, H.; Huffman, M.; Verma, D.; Pan, W.; Ho, H.-I.; Vroom, J.; Knight, A.; Hurtak, A.; Morris, W.; Strotman, N.; Murphy, G.; Maloney, K.; Fier, P. ChemRxiv. 2020, DOI: 10.26434/chemrxiv. 13472373.v1.

[6] Trimble, R. B.; Maley, F. J. Bacteriol. 1971, 108, 145-153.

[7] https://en.wikipedia.org/wiki/Cytidine_deaminase.

[8] Ferrero, M.; Gotor, V. Chem. Rev. 2000, 100, 12, 4319-4348.

[9] Romero-Tellez, S.; Lluch, J. M.; Gonzalez-Lafont, A.; Masgrau, L. Front. Chem. 2019, 7, 200.

[10] Moulis, C.; Guieysse, D.; Morel, S.; Severac, E.; Remaud-Simeon, M. Curr. Opin. Chem. Biol. 2021, 61, 96-106. 